ARTICLE

DOI: $10.1038 / s 41467-018-03172-5$

\title{
Directed gas phase formation of silicon dioxide and implications for the formation of interstellar silicates
}

Tao Yang (1) 1,2, Aaron M. Thomas ${ }^{1}$, Beni B. Dangi, ${ }^{1,3}$, Ralf I. Kaiser (1D ${ }^{1}$, Alexander M. Mebel ${ }^{4} \&$ Tom J. Millar (iD ${ }^{5}$

Interstellar silicates play a key role in star formation and in the origin of solar systems, but their synthetic routes have remained largely elusive so far. Here we demonstrate in a combined crossed molecular beam and computational study that silicon dioxide $\left(\mathrm{SiO}_{2}\right)$ along with silicon monoxide $(\mathrm{SiO})$ can be synthesized via the reaction of the silylidyne radical ( $\mathrm{SiH}$ ) with molecular oxygen $\left(\mathrm{O}_{2}\right)$ under single collision conditions. This mechanism may provide a low-temperature path-in addition to high-temperature routes to silicon oxides in circumstellar envelopes-possibly enabling the formation and growth of silicates in the interstellar medium necessary to offset the fast silicate destruction.

\footnotetext{
${ }^{1}$ Department of Chemistry, University of Hawai'i at Mānoa, Honolulu, HI 96822, USA. ${ }^{2}$ State Key Laboratory of Precision Spectroscopy, East China Normal University, Shanghai, 200062, China. ${ }^{3}$ Department of Chemistry, Florida Agricultural and Mechanical University, Tallahassee, FL 32307, USA. ${ }^{4}$ Department of Chemistry and Biochemistry, Florida International University, Miami, FL 33199, USA. ${ }^{5}$ Astrophysics Research Centre, School of Mathematics and Physics, Queen's University Belfast, Belfast, BT7 1NN, UK. Correspondence and requests for materials should be addressed to R.I.K. (email: ralfk@hawaii.edu) or to A.M.M. (email: mebela@fiu.edu) or to T.J.M. (email: tom.millar@qub.ac.uk)
} 
T he origin of interstellar silicate grains-nanoparticles consisting primarily of olivine-type $\left((\mathrm{Mg}, \mathrm{Fe})_{2} \mathrm{SiO}_{4}\right)$ refractory minerals-has remained a controversial topic for more than half a century, since interstellar silicates are faster destroyed by sputtering than formed during the late stages of stellar evolution through nucleation in circumstellar envelopes of oxygen-rich Asymptotic Giant Branch (AGB) and Red Supergiant (RSG) stars ${ }^{1-5}$. These nanoparticles have been associated with the prebiotic evolution of the interstellar medium (ISM) through the synthesis of molecular building blocks of life such as amino acids and sugars on their ice-coated surfaces by ionizing radiation ${ }^{6}$. Interstellar silicates also play a critical role in star formation and in the origin of solar systems contributing to the radiation balance and acting as a molecular feedstock, both through the formation of complex organics through the release of icy mantles that cover them and through disruption of grains in interstellar shocks ${ }^{3,7}$. In molecular clouds, they absorb light and hence shield complex organic molecules (COMs) - organics containing carbon, hydrogen, nitrogen, and oxygen like glycolaldehyde $\left(\mathrm{HCOCH}_{2} \mathrm{OH}\right)$ and formamide $\left(\mathrm{HCONH}_{2}\right)$-from the destructive interstellar ultraviolet radiation field ${ }^{8}$. Therefore, the elucidation of the origin of interstellar silicates is of vital importance to the astrochemistry, astrobiology, and astrophysics communities to eventually understand the fundamental processes that create a visible galaxy including our own.

A crucial point of concern is that the mass of dust ejected during the late stages of stellar evolution is produced at a rate that is significantly slower than the dust destruction time in the ISM, implying that grains also form in the lower density environment of the ISM ${ }^{5,9-14}$. Current astrochemical models of circumstellar envelopes propose that dust formation in AGB stars is driven eventually by clustering and reactions of silicon oxides along with magnesium-type and iron-type oxides ${ }^{2,15-20}$. There does appear to be, however, a severe discrepancy between the formation rates of silicate grains in circumstellar envelopes of $3 \times 10^{9}$ years and their destruction via sputtering once dispersed into the ISM that limits their lifetime to only a few $10^{8}$ years ${ }^{5,21-23}$. This discrepancy $^{24}$ may eventually be resolved through a better understanding of the processes of dust destruction ${ }^{5,23}$, but it remains possible that significant formation of dust needs to occur in the interstellar as opposed to the circumstellar medium ${ }^{12-15}$. Indeed, silicate grains may grow in the ISM by accreting and incorporating silicon oxide molecules ${ }^{25,26}$.

Here we show that the silicon dioxide molecule $\left(\mathrm{SiO}_{2}\right)$ along with silicon monoxide $(\mathrm{SiO})$ can be efficiently formed via a lowtemperature gas phase chemistry even at $10 \mathrm{~K}$. We report the results of a combined crossed molecular beam study and of electronic structure calculations on the reaction of the D1silylidyne radical $\left(\mathrm{SiD} ; \mathrm{X}^{2} \Pi\right)$ with molecular oxygen $\left(\mathrm{O}_{2}, \mathrm{X}^{3} \Sigma_{\mathrm{g}}{ }^{-}\right)$ leading to the formation of $\mathrm{SiO}_{2}$ and $\mathrm{SiO}$ through a barrierless reaction ${ }^{27}$. This system represents a proxy for the reaction of the silylidyne radical $(\mathrm{SiH})$ generated via photolysis of silane $\left(\mathrm{SiH}_{4}\right)$ 28-30 with $\mathrm{O}_{2}$ to synthesize silicon oxides via a single collision event. In the ISM, the reaction of $\mathrm{SiH}$ with $\mathrm{O}_{2}$ may represent a potential pathway to $\mathrm{SiO}_{2}$ and silicon oxide formation in those molecular clouds, where gas phase chemistry follows ice mantle sublimation; these silicon oxides might drive an exothermic chemistry that possibly produces larger silicon oxides ${ }^{25,31}$ leading ultimately to silicates at low temperatures.

\section{Results}

Crossed molecular beam studies in the laboratory frame. The reactive scattering experiments were performed using a crossed molecular beam apparatus (Methods). We monitored the scattering signal at mass-to-charge ratio $(\mathrm{m} / \mathrm{z}) 62\left({ }^{28} \mathrm{SiDO}_{2}{ }^{+}\right), 60$ $\left({ }^{28} \mathrm{SiO}_{2}{ }^{+}\right)$, and $44\left({ }^{28} \mathrm{SiO}^{+}\right)$. No signal was observed at $\mathrm{m} / \mathrm{z} 62$, suggesting that under single collision conditions the lifetime of the ${ }^{28} \mathrm{SiDO}_{2}$ adduct is shorter than its flight time to the electronimpact ionizer. Reactive scattering signal was detected at $\mathrm{m} / \mathrm{z} 60$ $\left({ }^{28} \mathrm{SiO}_{2}{ }^{+}\right)$(Fig. 1). The scattering signal is relatively weak, and at each angle up to $6 \times 10^{6}$ time-of-flight (TOF) spectra $(60 \mathrm{~h} \mathrm{col-}$ lection time) had to be averaged to obtain a reasonable signal-tonoise ratio. Signal detection at $\mathrm{m} / \mathrm{z} 60$ alone provides conclusive evidence on the formation of a molecule with the formula ${ }^{28} \mathrm{SiO}_{2}$ via a single collision event of two neutral reactants. Taking into account the data accumulation time, the signal-to-noise ratio obtained at $\mathrm{m} / \mathrm{z} 60$ and the abundances of naturally occurring silicon isotopes of ${ }^{30} \mathrm{Si}(3.10 \%),{ }^{29} \mathrm{Si}(4.67 \%)$, and ${ }^{28} \mathrm{Si}(92.23 \%)$, we would not expect-as confirmed experimentally-any reactive scattering signal at $m / z 62\left({ }^{30} \mathrm{SiO}_{2}{ }^{+}\right)$. Finally, the background counts at $\mathrm{m} / \mathrm{z} 44$ originating from singly ionized carbon dioxide $\left(\mathrm{CO}_{2}\right)$ in the detector precludes an identification of any reactive scattering signal at $\mathrm{m} / \mathrm{z} 44\left({ }^{28} \mathrm{SiO}^{+}\right)$. To summarize, the laboratory data indicate that a molecule with the formula ${ }^{28} \mathrm{SiO}_{2}$ (hereafter: $\mathrm{SiO}_{2}$ ) along with atomic deuterium is formed under single collision conditions in the reaction of the $\mathrm{SiD}$ radical with $\mathrm{O}_{2}$.

Crossed molecular beam studies in the center-of-mass frame. We transformed the experimental data from the laboratory to the center-of-mass $(\mathrm{CM})$ reference frame ${ }^{32}$ to gain information on the underlying reaction dynamics, which yields the CM translational energy flux distribution $P\left(E_{\mathrm{T}}\right)$ and the $\mathrm{CM}$ angular flux distribution $T(\theta)$ as depicted in Fig. 2. Best fits of the laboratory data are achieved with a single-channel fit forming products with a mass combination of $60 \mathrm{amu}\left(\mathrm{SiO}_{2}\right)$ and $2 \mathrm{amu}$ (D) (Figs. 1 and 2). A detailed inspection of the CM functions affords crucial information on the pertinent reaction channel(s) and dynamics. First, $P\left(E_{\mathrm{T}}\right)$ assists in the identification of the product isomer(s). For the reaction products formed without internal excitation, the high energy cutoff of $493 \pm 57 \mathrm{~kJ} \mathrm{~mol}^{-1}$ in $P\left(E_{\mathrm{T}}\right)$ denotes the sum of the absolute value of the reaction exothermicity plus the collision energy $E_{\mathrm{c}}\left(33.2 \pm 2.0 \mathrm{~kJ} \mathrm{~mol}^{-1}\right)$. A subtraction of the collision energy reveals that the reaction is highly exothermic with the energy of $-460 \pm 59 \mathrm{~kJ} \mathrm{~mol}^{-1}$. This finding agrees nicely with our computed value of $-441 \pm 5 \mathrm{~kJ} \mathrm{~mol}^{-1}$ (Fig. 3) and the energetics obtained from NIST Webbook $\left(-464 \mathrm{~kJ} \mathrm{~mol}^{-1}\right)^{33}$ to form the linear $\mathrm{SiO}_{2}$ molecule $\left(l-\mathrm{SiO}_{2}\right)$ along with atomic hydrogen $(\mathrm{H})$. This shows for the very first time that a $\mathrm{SiO}_{2}$ molecule is observed in the gas phase as a result of a reaction between two neutral species under controlled experimental conditions. Previously, Ahmed et al. generated $\mathrm{SiO}_{2}$ via laser ablation of silicon $(\mathrm{Si})$ and two successive oxygen abstractions from the seeding and reactant gas- $-\mathrm{CO}_{2},{ }^{34}$ Wang et al. formed gas phase $\mathrm{SiO}_{2}$ through $\mathrm{SiO}_{2}{ }^{-}$ electron photodetachment ${ }^{35}$. A complex forming reaction mechanism is evident from $T(\theta)$ which depicts the flux over the complete angular range ${ }^{36}$. This distribution reveals further a forward-backward symmetry proposing that the lifetime of the decomposing intermediate is longer than its rotational period ${ }^{37}$. Alternatively, a 'symmetric' reaction intermediate can account for these findings by emitting a deuterium atom with equal probabilities into $\theta$ and $\pi-\theta^{38}$.

Electronic structure calculations and reaction mechanism. We combined the experimental findings with electronic structure calculations on the reaction of $\mathrm{SiH}$ with $\mathrm{O}_{2}$ to elucidate the underlying dynamics (Fig. 3). These calculations were performed at a level of theory high enough to predict relative energies of the transition states and local minima as well as reaction energies within $5 \mathrm{~kJ} \mathrm{~mol}^{-1}$ (Methods). Based on the difference in zero 


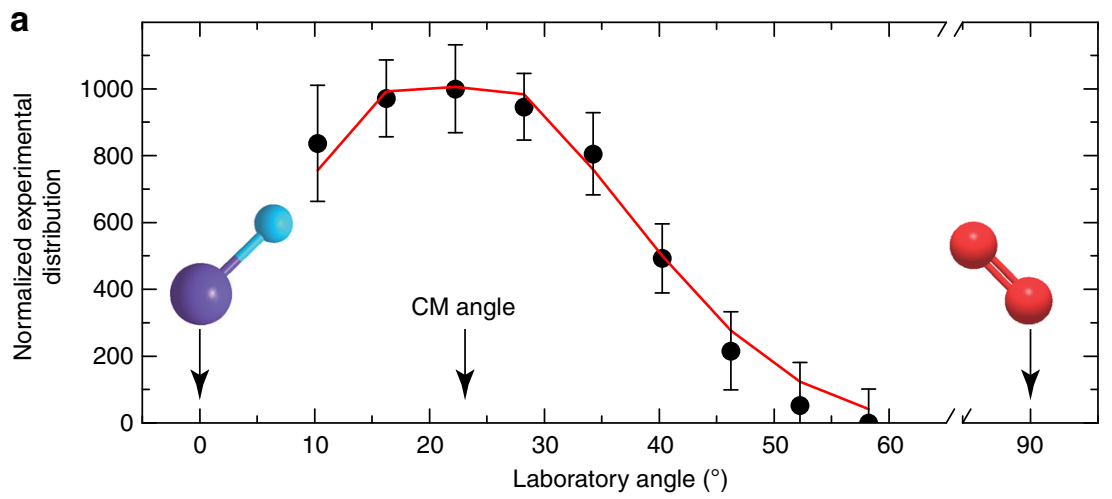

b

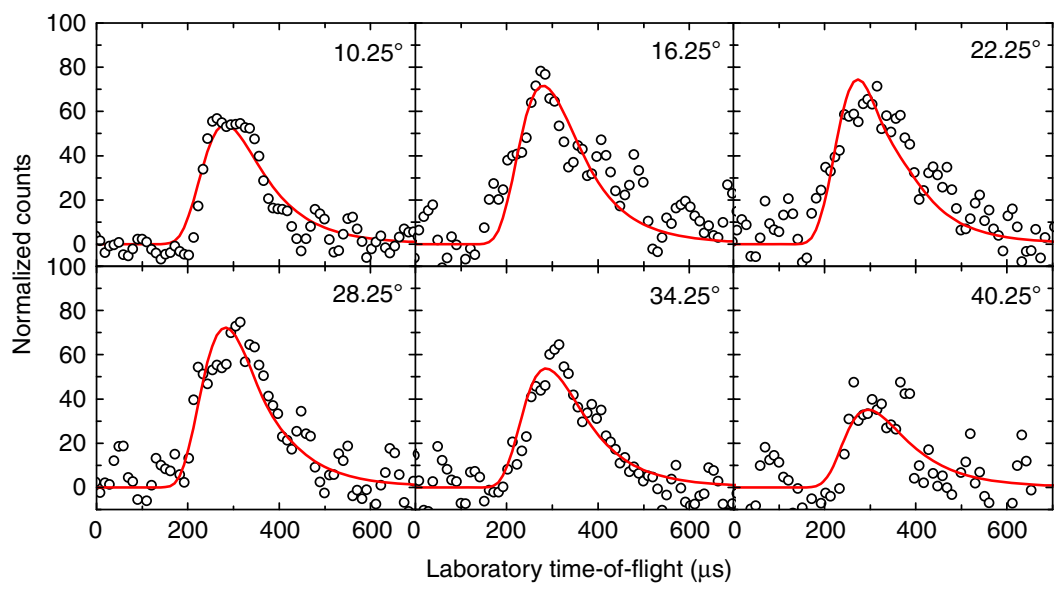

Fig. 1 Laboratory angular distribution and the associated time-of-flight spectra. Laboratory angular distribution at mass-to-charge ratio of $60\left(\mathrm{SiO}_{2}{ }^{+}\right.$) recorded in the reaction of the D1-silylidyne radical with molecular oxygen (a), and the time-of-flight spectra recorded at distinct laboratory angles overlaid with the best fits (b). The solid circles with their error bars indicate the normalized experimental distribution with $\pm 1 \sigma$ uncertainty (s.d. of the integrals of the time-of-flight spectra for the respective angle), and the open circles indicate the experimental data points of the time-of-flight spectra. The red lines represent the best fits obtained from the optimized center-of-mass (CM) functions, as depicted in Fig. 2
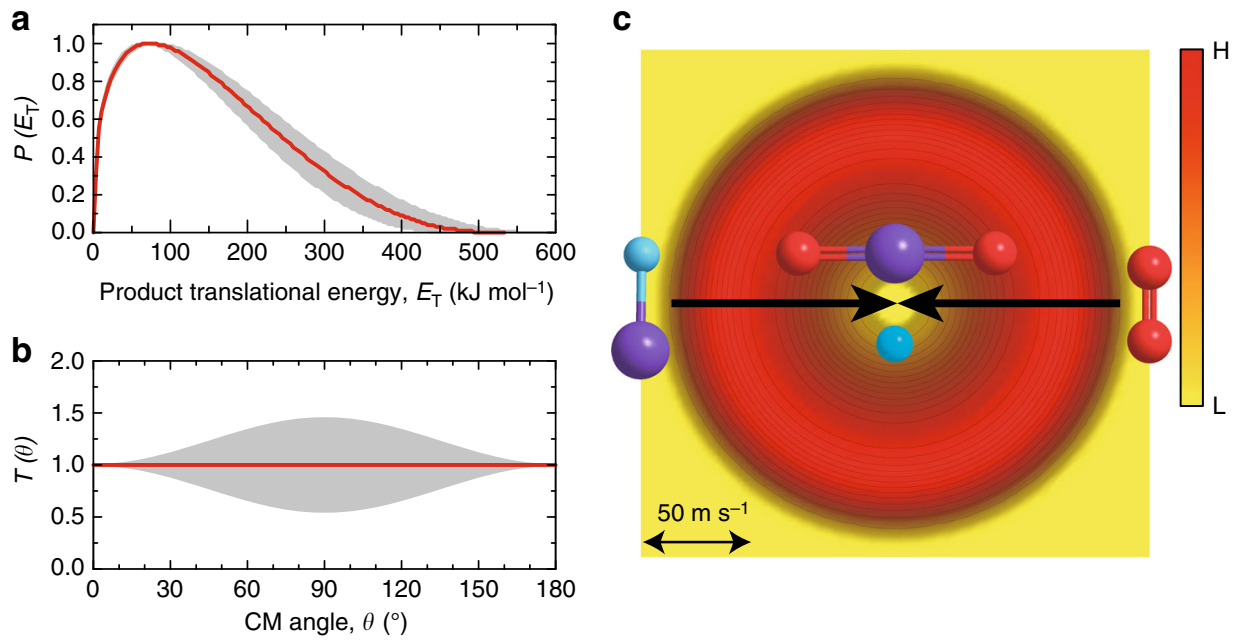

Fig. 2 Center-of-Mass (CM) distributions and the associated flux contour map. CM translational energy flux distribution (a), CM angular flux distribution (b), and the top view of their corresponding flux contour map (c) leading to the formation of silicon dioxide plus atomic deuterium in the reaction of D1silylidyne with molecular oxygen. Shaded areas indicate the error limits of the best fits accounting for the uncertainties of the laboratory angular distribution and TOF spectra, with the red solid lines defining the best-fit functions. The flux contour map represents the flux intensity of the reactive scattering products as a function of the $\mathrm{CM}$ scattering angle $(\theta)$ and product velocity $(u)$. The color bar indicates the flux gradient from high $(\mathrm{H})$ intensity to low (L) intensity. Colors of the atoms: silicon (purple), oxygen (red), and deuterium (light blue) 


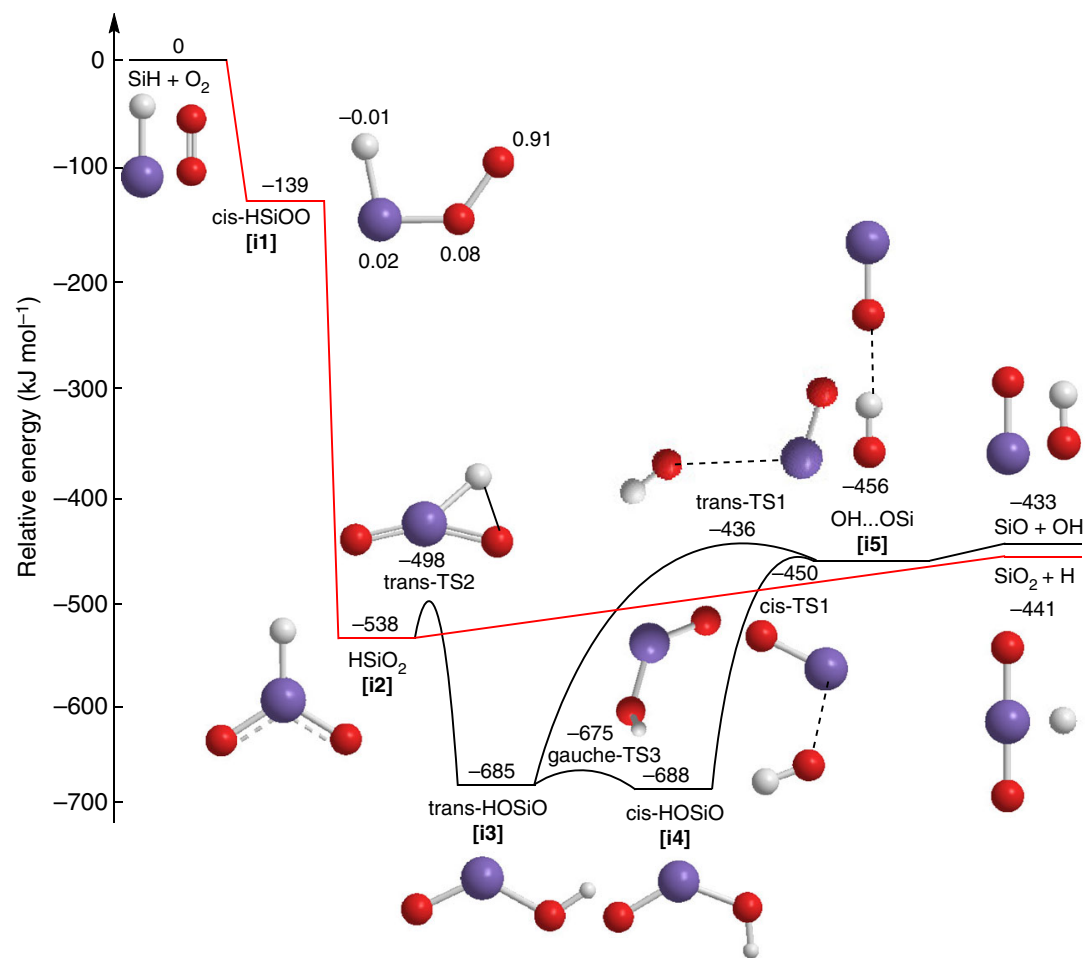

Fig. 3 Potential energy surface. The potential energy surface for the reaction of the silylidyne radical with molecular oxygen including reaction pathways energetically accessible in the crossed molecular beam experiments. The route in red highlights the reaction pathway leading to the formation of silicon dioxide plus atomic hydrogen. Relative energies are given in units of $\mathrm{kJ} \mathrm{mol}^{-1}$. Note that the relative energy of silicon dioxide plus atomic deuterium is $3 \mathrm{~kJ}$ $\mathrm{mol}^{-1}$ higher as compared to non-deuterated reactants, whereas for the intermediates and transition states in the $\mathrm{SiD}-\mathrm{O}_{2}$ and $\mathrm{SiH}-\mathrm{O}_{2}$ systems, relative energies are within 1 to $2 \mathrm{~kJ} \mathrm{~mol}^{-1}$. For [i1], the spin density distribution is also shown. Colors of the atoms: silicon (purple), oxygen (red), and hydrogen (light grey)

point vibrational energies by replacing hydrogen $(\mathrm{H})$ with deuterium (D) in $\mathrm{SiH}$, the relative energy of $\mathrm{SiO}_{2}$ plus $\mathrm{D}$ is $3 \mathrm{~kJ} \mathrm{~mol}^{-1}$ higher than that of non-deuterated reactants, whereas for the intermediates and transition states in the $\mathrm{SiD}-\mathrm{O}_{2}$ and $\mathrm{SiH}-\mathrm{O}_{2}$ systems, which maintain the $\mathrm{Si}-\mathrm{H}(\mathrm{D})$ bond intact, relative energies are within 1 to $2 \mathrm{~kJ} \mathrm{~mol}^{-1}$. The computations verify the experimental results of an indirect reaction mechanism. Here, the reaction is initiated by a barrierless addition of $\mathrm{SiH}$ with its radical center to $\mathrm{O}_{2}$ at a single oxygen atom yielding a $\mathrm{C}_{\mathrm{s}}$ symmetric cis-HSiOO intermediate [i1] on the doublet surface. The barrierless addition was verified by a careful examination of the entrance channel, which indicates that the potential energy of the system monotonically decreases as $\mathrm{SiH}$ approaches $\mathrm{O}_{2}$. The collision complex [i1] is only metastable and undergoes a rapid atomic oxygen migration to the silicon atom forming the $\mathrm{C}_{2 \mathrm{v}}$ symmetric $\mathrm{HSiO}_{2}\left({ }^{2} \mathrm{~B}_{1}\right)$ intermediate [i2], which is strongly bound by $538 \mathrm{~kJ} \mathrm{~mol}^{-1}$ with respect to the separated reactants. This intermediate can either undergo unimolecular decomposition via a loose exit transition state by $\mathrm{H}$ loss forming $\mathrm{SiO}_{2}\left({ }^{1} \Sigma_{\mathrm{g}}{ }^{+}\right)$ or isomerize via hydrogen migration to trans-HOSiO $\left(\mathrm{C}_{\mathrm{s}},{ }^{2} \mathrm{~A}\right.$, [i3]), which in turn undergoes trans-cis isomerization to cisHOSiO $\left(\mathrm{C}_{\mathrm{s}},{ }^{2} \mathrm{~A}\right.$, , [i4]). Multireference CASPT2 calculations with full active spaces $(17,13)$ corroborate the conclusions that the reversed addition reaction of $\mathrm{H}$ to $\mathrm{SiO}_{2}$ is barrierless. The trans$\mathrm{HOSiO}$ and cis-HOSiO intermediates are isovalent to the wellknown trans-HOCO and cis-HOCO intermediates, reside in deep potential energy wells of 685 and $688 \mathrm{~kJ} \mathrm{~mol}^{-1}$, and can undergo facile $\mathrm{Si}-\mathrm{O}$ bond cleavages through loose exit transition states yielding a linear van-der-Waals complex between the hydroxyl radical $(\mathrm{OH})$ and $\mathrm{SiO}$ [i5], in which $\mathrm{OH}$ is hydrogen bridge bonded to the oxygen atom of $\mathrm{SiO}$. This complex is bound by 23 $\mathrm{kJ} \mathrm{mol}^{-1}$ with respect to the separated products. Overall, the computations revealed two competing exit channels: the formation of $\mathrm{SiO}_{2}$ plus $\mathrm{H}$ and $\mathrm{SiO}$ plus $\mathrm{OH}$. With the exception of [i1], the aforementioned energetics are within $13 \mathrm{~kJ} \mathrm{~mol}^{-1}$ when compared with Schaefer's study on the stationary points relevant to the reaction of $\mathrm{SiO}$ with $\mathrm{OH} ;{ }^{39}$ Darling and Schlegel predicted the existence of [i1], but their energetics, computed at the G2 level of theory, forecasted the energy difference between [i1] and [i4] to be about $505 \mathrm{~kJ} \mathrm{~mol}^{-1}$ compared to $549 \mathrm{~kJ} \mathrm{~mol}^{-1}$ in our system ${ }^{40}$. Finally, since our experimental setup could not probe the $\mathrm{SiO}$ route, the branching ratios were determined computationally exploiting Rice-Ramsperger-Kassel-Marcus (RRKM) theory (Methods). The relative yields of $\mathrm{SiO}_{2}$ and $\mathrm{SiO}$ were virtually independent of the collision energy between 0 and $36 \mathrm{~kJ}$ $\mathrm{mol}^{-1}$ and varied in the ranges of $49.5 \pm 2.5$ and $50.5 \pm 2.5 \%$. We should note, however, that the energy content in the intermediates [i1] to [i5] is so significant that the RRKM rate constants are close to the applicability of the statistical theory. Therefore, dynamical effects might affect the branching ratios, but a $50-50 \%$ partition is reasonable given the closeness of the reaction energies.

\section{Discussion}

Let us first address the barrierless character of the reaction of $\mathrm{SiH}$ with $\mathrm{O}_{2}$. The initial addition step to [i1] has no barrier because it represents an association of two species, each of them having at least one unpaired electron, in this case a radical $(\mathrm{SiH})$ and a diradical $\left(\mathrm{O}_{2}\right)$. During the association process two unpaired electrons from the two interacting moieties form an electron pair thus creating a new $\mathrm{Si}-\mathrm{O}$ single bond. Such radical/(di)radical reactions occur without barriers. While the complex [i1] is formed, the total spin of the doublet/triplet pair is converted to a doublet due to the formation of the extra electron pair. The 
calculated spin density distribution in [i1] shows that the remaining unpaired electron in $\mathrm{HSiOO}$ is localized on the terminal oxygen atom, which exhibits a spin density of 0.91 (Fig. 3). It is interesting to note that the related reactions of $\mathrm{Si}$ with $\mathrm{O}_{2}$ (diradical/diradical) and singly ionized silicon $\left(\mathrm{Si}^{+}\right)$with $\mathrm{O}_{2}$ (radical/diradical) are also barrierless ${ }^{41,42}$. However, these reactions cannot produce $\mathrm{SiO}_{2}$ or singly ionized silicon dioxide $\left(\mathrm{SiO}_{2}{ }^{+}\right)$under single collision conditions as prevailing in the low density ISM. Here, $\mathrm{SiO}_{2}$ and $\mathrm{SiO}_{2}{ }^{+}$represent highly exothermic reaction intermediates residing in deep potential wells, and their internal energy due to chemical activation has to be dissipated. The energy dissipation may occur either via deactivation through third-body collisions or via fragmentation to $\mathrm{SiO}$ plus $\mathrm{O}$ or singly ionized silicon monoxide $\left(\mathrm{SiO}^{+}\right)$plus $\mathrm{O}$, respectively. Whereas third-body collisions prevent the dissociation of $\mathrm{SiO}_{2}$ when the reaction of $\mathrm{Si}$ with $\mathrm{O}_{2}$ takes place in a $0.37 \mathrm{~K}$ superfluid helium droplet $^{43}$, the fragmentation channels take over at low pressures where collision deactivations are inefficient. Indeed, the reaction of $\mathrm{Si}$ with $\mathrm{O}_{2}$ is usually invoked as one of the main source of $\mathrm{SiO}^{44}$. Crossed molecular beam studies of this system have firmly established $\mathrm{SiO}$ plus $\mathrm{O}$ as the reaction products under nearly zero-pressure conditions ${ }^{45}$ as corroborated by theoretical quasiclassical trajectory calculations ${ }^{46}$. An alternative reaction leading to the formation of $\mathrm{SiO}_{2}$ plus $\mathrm{O}$, i.e., the reaction of $\mathrm{SiO}$ with $\mathrm{O}_{2}$, which plays an important role in fabrication of silicon oxide films at elevated temperatures ${ }^{47}$, is known to be endothermic by $28 \mathrm{~kJ}$ $\mathrm{mol}^{-1}$ and has a barrier of at least $91 \mathrm{~kJ} \mathrm{~mol}^{-1}$ and hence cannot occur in low-temperature interstellar environments ${ }^{48}$.

The $\mathrm{SiH}$ reaction with $\mathrm{O}_{2}$ is akin to reaction of the isovalent methylidyne radical $(\mathrm{CH})$ with $\mathrm{O}_{2}$, which is also known to be barrierless and consequently very fast at the collisional kinetic limit even at extremely low temperatures such as $13 \mathrm{~K}^{49}$. Theoretically, low-temperature rate coefficients for this system have been evaluated using long-range transition state theory and the calculations reproduced the experimental values within a factor of two to three ${ }^{50}$. Here we exploited the same theoretical method to compute rate coefficients for the $\mathrm{SiH}-\mathrm{O}_{2}$ reaction and obtained values slightly increasing from $3.1 \times 10^{-10} \mathrm{~cm}^{3}$ molecule $\mathrm{s}^{-1}$ at $10 \mathrm{~K}$ to $4.4 \times 10^{-10}$ and $5.3 \times 10^{-10} \mathrm{~cm}^{3}$ molecule $\mathrm{e}^{-1} \mathrm{~s}^{-1}$ at 100 and $300 \mathrm{~K}$, respectively, compared to an experimental value of $1.7 \times 10$ $-10 \mathrm{~cm}^{3}$ molecule $\mathrm{s}^{-1}$ at $298 \mathrm{~K}^{51}$. A comparison of the rate coefficients at $13 \mathrm{~K}$ for the $\mathrm{CH}-\mathrm{O}_{2}\left(1.5 \times 10^{-10} \mathrm{~cm}^{3}\right.$ molecule ${ }^{-1} \mathrm{~s}^{-1}$ (experiment) and $2.9 \times 10^{-10} \mathrm{~cm}^{3}$ molecule $\mathrm{s}^{-1} \mathrm{~s}^{-1}$ (theory)) and $\mathrm{SiH}-\mathrm{O}_{2}\left(3.2 \times 10^{-10} \mathrm{~cm}^{3}\right.$ molecule ${ }^{-1} \mathrm{~s}^{-1}$ (theory)) reveals that both reactions should be nearly equally fast. The calculations also demonstrate that the long-range $\mathrm{SiH} / \mathrm{O}_{2}$ interaction is dominated by dispersion forces, with small contributions from dipole $(\mathrm{SiH})$ -induced dipole $\left(\mathrm{O}_{2}\right)$ and dipole $(\mathrm{SiH})$-quadrupole $\left(\mathrm{O}_{2}\right)$ interactions.

Having established the formation of $\mathrm{SiO}_{2}$ along with $\mathrm{SiO}$ under single collision conditions in the laboratory and through electronic structure calculations, we now discuss potential astrochemical implications. It is essential to transfer these findings to 'real' extraterrestrial environments since all experiments conducted under well-defined laboratory conditions can hardly mimic the chemical complexity of the ISM, where both neutral-neutral and ion-molecule reactions along with photochemical processes occur simultaneously $6,38,52$. Our studies indicate unambiguously that the reaction has no entrance barrier, all barriers involved in the formation of the silicon oxides are well below the energy of the separated reactants, and the overall reactions to form the silicon oxides are exothermic. These findings represent a crucial prerequisite for this reaction to be important in low-temperature molecular clouds; any barrier would block these reactions in low-temperature interstellar environments. Therefore, our results can be universally applied to any cold interstellar environment such as molecular clouds, where ice mantles can be removed from grains, and where adequate concentrations of $\mathrm{SiH}$ radicals and $\mathrm{O}_{2}$ exist.

In cold molecular clouds, $\mathrm{O}_{2}$ is difficult to detect. This is in part due to the fact that this diatomic molecule is homopolar, and that observations are best made from space platforms such as Herschel ${ }^{53}$. The former implies that large concentrations (or column densities) are needed for detection, the latter that small telescope diameters and large beam sizes dilute the signal intensity thus making molecular oxygen difficult to detect even in sources such as hot molecular cores. Hence, the recent detection of $\mathrm{O}_{2}$ toward Orion and $\rho$ Ophiuchi $A$ is truly extraordinary ${ }^{53-56}$. Gas phase abundances of $\mathrm{O}_{2}$ with respect to molecular hydrogen $\left(\mathrm{H}_{2}\right)$ at the order of $10^{-7}-10^{-6}$ would be in good agreement with astronomical observations ${ }^{53,57}$, however, they are still significantly lower than the upper limit to the $\mathrm{O}_{2}$ abundance in interstellar ice. Due to its non-polar nature, upper limits to the abundance of $\mathrm{O}_{2}$ in interstellar ice are difficult but its effect on the carbon monoxide (CO) vibrational absorption band at $4.673 \mu \mathrm{m}\left(2140 \mathrm{~cm}^{-1}\right)$ is consistent with ice mixtures in which $\mathrm{O}_{2}$ is comparable to or a few times more abundant than $\mathrm{CO}^{57}$. Vandenbussche et al. (1999) provided upper limits ${ }^{58}$ on the $\mathrm{O}_{2}$ ice abundance based on the non-detection of its fundamental vibrational band in the solid state, at $6.45 \mu \mathrm{m}\left(1550 \mathrm{~cm}^{-1}\right)$, as well as on observations of the $4.673 \mu \mathrm{m}\left(2140 \mathrm{~cm}^{-1}\right) \mathrm{CO}$ band. Toward the protostar R CrA IRS2, they find an upper limit of $50 \%$ with respect to solid CO and, in NGC7538 IRS9, an upper limit of $20 \%$ to water ice which has an estimated abundance of $10^{-4}$ with respect to $\mathrm{H}_{2}$. In general, the few sources in which observations have been carried out are consistent with upper limits to the $\mathrm{O}_{2}$ ice abundances in the range $10^{-5}-10^{-4}$ with respect to $\mathrm{H}_{2}$. This abundance is consistent with $\mathrm{O}_{2}$ to water ratio $(0.038)$ recently detected in comet $67 \mathrm{P} / \mathrm{Churyumov}-\mathrm{Gerasimenko}{ }^{59}$. Indeed, Taquet et al. argued that the high abundance of $\mathrm{O}_{2}$ as seen in this $67 \mathrm{P}$ is of interstellar origin ${ }^{60}$. Further, we should note that the abundance of $\mathrm{SiH}$ depends on the removal of the ice mantles. Removal provides a parent species (silane; $\mathrm{SiH}_{4}$ ) to the gas phase with subsequent photodissociation producing $\mathrm{SiH}^{28-30}$, a molecule with a very small dipole moment of only 0.12 Debye $^{61}$. Gas phase detection has therefore been difficult with only a tentative identification to date in Orion ${ }^{62}$. While multiple reaction pathways can lead to $\mathrm{SiO}_{2}$, the neutral-neutral reaction of $\mathrm{SiH}$ with $\mathrm{O}_{2}$ has a universal potential to synthesize $\mathrm{SiO}_{2}$ along with $\mathrm{SiO}$ in cold molecular clouds when the fractional abundance of $\mathrm{O}_{2}$ in the gas phase is sufficient. Both $\mathrm{SiO}_{2}$ and $\mathrm{SiO}$-as derived from laboratory studies of barrier-less condensation of silicon oxides in helium droplet experiments ${ }^{25,43}$ and theoretical chemistry calculations that show reactions between these oxides produce larger silicon oxides in exothermic chemistry ${ }^{16,17,31}$ - may eventually play a role in the formation and growth of interstellar silicates. It should be noted that the elementary reactions leading to small silicon oxides may also be relevant to the chemistry of silicon oxide plasmas, such as those widely used in the semiconductor industry for depositing thin film insulators in integrated circuits like memory or processor chips ${ }^{63}$. During the $\mathrm{Si}$ and $\mathrm{SiO}_{2}$ chemical vapor deposition (CVD) processes, primary precursors such as silane or disilane $\left(\mathrm{Si}_{2} \mathrm{H}_{6}\right)$ undergo bond cleavage processes with the fragments adsorbing on the surfaces of the substrates, during which complex gas-phase and gas-surface reactions are involved ${ }^{64}$. Layered films of silicon oxides can be processed by mixing silane or disilane with $\mathrm{O}_{2}$ and dinitrogen monoxide $\left(\mathrm{N}_{2} \mathrm{O}\right)$, for instance ${ }^{65}$. Here depositions at low temperatures are preferred, since high-temperature instabilities on the substrates can substantially reduce the film production rates ${ }^{66}$. Plasma-enhanced CVD (PECVD) represents the main processing route for silicon oxide film growth; here, a radio 
frequency (RF) discharge supplies energy to initiate bond rupture processes and hence to promote the chemical reaction ${ }^{67,68}$. Since silicon hydride radical species such as $\mathrm{SiH}_{\mathrm{m}}$ and $\mathrm{Si}_{2} \mathrm{H}_{\mathrm{n}}(\mathrm{m}=1-3$, $\mathrm{n}=1-5$ ) are generated as well, PECVD is extraordinarily complex ${ }^{64,69}$. Therefore, it is vital to untangle the reaction mechanisms from the fundamental, microscopic point of view.

Our combined crossed molecular beam and electronic structure calculations provide compelling evidence on the formation of $\mathrm{SiO}_{2}$ along with $\mathrm{SiO}$ under single collision conditions. The bimolecular neutral-neutral reaction of $\mathrm{SiH}$ with $\mathrm{O}_{2}$ represents a single step mechanism to form two silicon oxides- $\mathrm{SiO}$ and $\mathrm{SiO}_{2}$. In combination with astrochemical modeling, our study suggests that silicon oxides may form not only in hot circumstellar envelopes of oxygen-rich stars as thought previously, but also in interstellar clouds via facile, barrierless reactions involving the simplest silicon-bearing radical $(\mathrm{SiH})$ and $\mathrm{O}_{2}$. These pathways provide a population of silicon oxides, which can possibly provide a basis for the regeneration of interstellar silicates thus leading us closer to solving the paradox of the injection and destruction timescales of silicates ${ }^{5,21-24}$. The ability of barrierless, exothermic reactions between $\mathrm{SiO}$ and $\mathrm{SiO}_{2}$ to form larger silicon oxides such as $\mathrm{Si}_{2} \mathrm{O}_{5}$ and $\mathrm{Si}_{3} \mathrm{O}_{5}$ suggests that such reactions ${ }^{31}$ may play a central role in the process by which reformation of silicate grains must occur in the ISM.

Although there is no detailed description to date of how silicate dust grains might form in the ISM, silicon oxides are likely involved. Therefore, the work reported here represents an important step toward a systematic understanding of the fundamental chemical processes eventually leading to the formation of silicate grains in the ISM. Since distinct types of interstellar grains (silicates, carbonaceous grains, and silicon carbide) exist, the current work resembles a template for future studies of elementary chemical reactions relevant to grain formation. This requires a sophisticated link of laboratory, theoretical, and modeling study with astronomical observations, particularly for those highdensity regions in circumstellar envelopes, hot molecular cores, shocked gas, including supernova remnants. Considering the crucial role of interstellar dust in star and hence solar system formation ${ }^{70}$ and in the chemical evolution of the universe, with grains providing critical molecular factories to even synthesize bio-relevant organics like amino acids and sugars ${ }^{7}$, the unraveling of the cosmic dust enigma is of fundamental importance to the understanding of our origins.

\section{Methods}

Crossed molecular beam experiments. The experiments were conducted in a crossed molecular beam machine under single-collision conditions at the University of Hawai'i at Mānoa ${ }^{71,72}$. In the primary reactant chamber, a pulsed and supersonic beam of the D1-silylidyne radical $\left(\mathrm{SiD} ; \mathrm{X}^{2} \Pi\right)$ at fractions of about $0.5 \%$ was prepared in situ by ablation of a rotating silicon rod with the output from a Spectra-Physics Quanta-Ray Pro 270 Nd:YAG laser $(30 \mathrm{~Hz}, 266 \mathrm{~nm}, 10-15 \mathrm{~mJ}$ pulse energies), with the ablated species further entrained by molecular deuterium $\left(D_{2}, 99.7 \%\right.$; Icon Isotopes, Inc.). The $\mathrm{D}_{2}$ gas acts as a carrier and reactant gas, and no other silicon-deuterium-bearing molecules were found to be present in the beam under the experimental conditions. Considering the natural isotope abundances of silicon, it was easier to optimize a SiD beam at $\mathrm{m} / z$ 31. The molecular beam entraining the $\mathrm{SiD}$ radicals then passed a skimmer and a chopper wheel, generating a pulsed radical beam of a well-defined peak velocity of $1981 \pm 38 \mathrm{~m} \mathrm{~s}^{-1}$ and speed ratio of $5.2 \pm 1.0$. Notice that even if $\mathrm{SiD}$ radicals of $\mathrm{A}^{2} \Delta$ state were formed, they can decay to the ground state during the travel time of about $18 \mu$ s to the interaction region of the scattering chamber ${ }^{73}$. In the scattering chamber, this segment crossed a supersonic beam of pure oxygen gas $\left(\mathrm{O}_{2} ; 99.998 \%\right.$; Matheson) perpendicularly, which had a velocity of $778 \pm 20 \mathrm{~m} \mathrm{~s}^{-1}$ and a speed ratio of $15.6 \pm$ 1.0. This setup eventually yielded a collision energy of $33.2 \pm 2.0 \mathrm{~kJ} \mathrm{~mol}^{-1}$ and a center-of-mass $(\mathrm{CM})$ angle of $23.3 \pm 1.2^{\circ}$. The reactive scattering products were ionized by an electron-impact ionizer operating at $80 \mathrm{eV}$ and $2 \mathrm{~mA}$ emission current, before they entered a quadrupole mass spectrometer (QMS, Extrel QC $150)$ operating in the time-of-flight (TOF) mode. The selected ion species filtered by the QMS at a specific $m / z$ travelled towards a stainless steel target coated with a thin layer of aluminum biased at $-22.5 \mathrm{kV}$ and a cascade-of-electron pulse was initiated upon impact. The electrons were then expelled from the stainless steel target and flew toward an organic scintillator to generate a photon pulse, prior to be detected by a Burle photomultiplier tube (PMT, Model 8850) operating at -1 $35 \mathrm{kV}$. The signal was eventually filtered by a discriminator (Advanced Research Instruments, Model F-100TD) at a level of $1.6 \mathrm{mV}$ before being fed into a Stanford Research System SR430 multichannel scaler. The whole detection region is housed in a triply, differentially pumped vacuum chamber, which can be rotated in a plane defined by the primary and secondary beams, thus we are able to record TOF spectra at discrete angles, integrate and normalize them with respect to the intensity at the CM angle, and then extract the product laboratory angular distribution at a specific $m / z^{74}$. In order to obtain the information about the reaction dynamics, we employed a forward-convolution routine based on the Jacobian transformation to convert the data in the laboratory frame into the $\mathrm{CM}$ frame $\mathrm{e}^{32,75}$ This method actually begins with a trial set of parameterized CM functions-the translational energy flux distribution, $P\left(E_{\mathrm{T}}\right)$, and the angular flux distribution, $T(\theta)$, in the CM frame, to iteratively fit the laboratory TOF spectra and the angular distribution until the best fits are reached, accounting for apparatus performances, beam divergences, and velocity spreads. We can then plot a flux contour map, $I(\theta$ $u)=P(u) \times T(\theta)$, which presents the flux of the reactive scattering products as a function of the CM scattering angle $(\theta)$ and product velocity $(u)$, and reveals information on the scattering reaction dynamics ${ }^{76}$.

Electronic structure calculations. Geometries of most of the intermediates, transition states, and bimolecular fragments were adapted from Schaefer et al. ${ }^{39}$ where they were optimized at the $\operatorname{CCSD}(\mathrm{T}) / / \mathrm{cc}-\mathrm{pV}(\mathrm{Q}+\mathrm{d}) Z$ level of theory. Singlepoint energies were computed using the explicitly correlated $\operatorname{CCSD}(\mathrm{T})-\mathrm{F} 12$ method $^{77}$ with the correlation-consistent aug-cc-pV5Z basis set; ${ }^{78,79}$ this theoretical approach closely approximates $\operatorname{CCSD}(\mathrm{T})$ energies at the complete basis set (CBS) limit and is expected to provide relative energies with an accuracy of $5 \mathrm{~kJ} \mathrm{~mol}$ ${ }^{-1}$. In addition to the structures considered by Schaefer et al. ${ }^{39}$, we searched for cis$\mathrm{HSiOO}$ and trans-HSiOO and cyclic $\mathrm{HSiO}_{2}$ intermediates, which may represent initial complexes produced in the reaction of $\mathrm{SiH}$ with $\mathrm{O}_{2}$. We employed the same $\operatorname{CCSD}(\mathrm{T}) / / \mathrm{cc}-\mathrm{pV}(\mathrm{Q}+\mathrm{d}) Z$ method for their geometry optimization. Only the cis$\mathrm{HSiOO}$ structure [i1] was located as a local minimum on the potential energy surface. A trans-HSiOO structure spontaneously converges to the $\mathrm{HSiO}_{2}$ isomer [i2] during optimization. A cyclic $\mathrm{HSiO}_{2}$ geometry optimizes to a stationary point within $\mathrm{C}_{2 \mathrm{v}}$ symmetry, but shows one imaginary frequency. Once symmetry is released, the further optimization yields the open $\mathrm{HSiO}_{2}$ isomer [i2]. Next, we searched for a transition state connecting cis-HSiOO [i1] with $\mathrm{HSiO}_{2}$ [i2]. The saddle-point optimization gave a non-planar structure typical for a transition state for rotation around the $\mathrm{Si}-\mathrm{O}$ bond. The transition state would normally connect two planar cis-HSiOO and trans-HSiOO minima, but since the latter spontaneously rearranges to $\mathrm{HSiO}_{2}$, this transition state is in fact between [i1] and [i2]. Furthermore, the transition state appears to be only slightly higher in energy than [i1] at the $\operatorname{CCSD}(\mathrm{T}) / / \mathrm{cc}-\mathrm{pV}(\mathrm{Q}+\mathrm{d}) Z$ level, and becomes lower in energy than [i1] when $\mathrm{ZPE}$ corrections are included. The transition state energy is below that of cis$\mathrm{HSiOO}$ at our final CCSD(T)-F12/aug-cc-pV5Z $+\mathrm{ZPE}(\mathrm{CCSD}(\mathrm{T}) / \mathrm{cc}-\mathrm{pV}(\mathrm{Q}+\mathrm{d}) \mathrm{Z})$ level of theory. This result indicates that [i1] could be only a metastable structure on the potential energy surface, which rapidly isomerizes to $\mathrm{HSiO}_{2}$ [i2]. In this view, the entrance channel of the $\mathrm{SiH}+\mathrm{O}_{2}$ reaction can be described as addition of $\mathrm{O}_{2}$ to the radical site on the $\mathrm{Si}$ atom either in cis-conformation or trans-conformation followed by spontaneous rearrangement to $\mathrm{HSiO}_{2}$ resulting in formal insertion of HSi into the $\mathrm{O}=\mathrm{O}$ bond of $\mathrm{O}_{2}$. The process is driven by its very-high exothermic energy of $538 \mathrm{~kJ} \mathrm{~mol}^{-1}$. We have also carefully mapped out the potential energy surface for $\mathrm{H}$ loss from $\mathrm{HSiO}_{2}$ [i2] to verify that this process occurs without an exit barrier. Within $\mathrm{C}_{2 \mathrm{v}}$ symmetry, the electronic state of [i2] is ${ }^{2} \mathrm{~B}_{2}$ but the products, $\mathrm{SiO}_{2}+\mathrm{H}$, correlate to the ${ }^{2} \mathrm{~A}_{1}$ state. Therefore, the $\mathrm{H}$ loss is a symmetry-forbidden process within the $\mathrm{C}_{2 \mathrm{v}}$ point group. If symmetry is reduced to $\mathrm{C}_{\mathrm{s}}$, the two states involved are both ${ }^{2} \mathrm{~A}^{\prime}$ and hence $\mathrm{H}$ elimination should take place via a crossing or avoided crossing of the two ${ }^{2} \mathrm{~A}^{\prime}$ states. Therefore, we scanned the minimal energy path for the $\mathrm{H}$ loss from [i2] using the multireference secondorder perturbation theory CASPT2 method ${ }^{80}$ with the full valence active space containing 17 electrons distributed on 13 orbitals $(17,13)$ with the aug-cc-pVDZ basis set. The CASPT2 $(17,13) /$ aug-cc-pVDZ geometry optimizations and vibrational frequency calculations were carried out for various $\mathrm{Si}-\mathrm{H}$ distances frozen at the values from $1.5 \AA$ to $4.0 \AA$ with a step of $0.2 \AA$. Following these geometry optimizations, relative energies of the MEP structures were refined by employing CASPT2 $(17,13)$ calculations with a larger aug-cc-pVTZ basis set and with mixing wave functions of the two lowest ${ }^{2} \mathrm{~A}$ ' involved in the process. The MEP has shown a steady energy decrease with the $\mathrm{Si}-\mathrm{H}$ distance from [i2] to the $\mathrm{SiO}_{2}+\mathrm{H}$ products confirming the barrierless character of the $\mathrm{H}$ elimination. All CCSD(T), CCSD(T) F12, and CASPT2 calculations in this work were performed using the MOLPRO 2010 program package ${ }^{81}$. Collision-energy dependent rate constants for chemically activated unimolecular reactions on the $\mathrm{HSiO}_{2}$ surface starting from the intermediate [i2] were carried out employing RRKM theory using the in-house code ${ }^{82}$ The internal energy of each intermediate was taken as a sum of its chemical activation energy (the negative of its relative energy with respect to the $\mathrm{SiH}+\mathrm{O}_{2}$ reactants) plus the collision energy. For reaction steps proceeding well-defined transition states, we used CCSD(T)-F12/aug-cc-pV5Z relative energies calculated here and $\operatorname{CCSD}(\mathrm{T}) / \mathrm{cc}-\mathrm{pV}(\mathrm{Q}+\mathrm{d}) Z$ vibrational frequencies reported by Schaefer 
et al. ${ }^{39}$ For the [i2] $\rightarrow \mathrm{SiO}_{2}+\mathrm{H}$ barrierless reaction step, we employed variational transition state theory (VTST) ${ }^{83,84}$ with transition state candidate structures ranging along the MEP. Here we used vibrational frequencies of the MEP structures calculated at the CASPT2 $(17,13) /$ aug-cc-pVDZ level and their relative energies obtained at CASPT2 $(\mathrm{mix}=2,17,13)$ /aug-cc-pVTZ. For barrierless dissociation of the $\mathrm{OH}$...OSi complex [i5] to the $\mathrm{SiO}+\mathrm{OH}$ products, we also utilized the VTST approach. Here, the wave function is single-reference, the interaction has a van der Waals character, and hence the MEP was mapped out using a density functional wB97XD method ${ }^{85}$ including a dispersion correction with the aug-cc-pVTZ basis set. Single-point relative energies of the MEP structures were refined at our best CCSD(T)-F12/aug-cc-pV5Z level and were used in VTST calculations in conjunction with wB97XD/aug-cc-pVTZ frequencies. The dipole and quadrupole moments and polarizabilities of $\mathrm{SiH}$ and $\mathrm{O}_{2}$ required for the calculations of the capture rate coefficients using long-range transition state theory were also computed at the wB97XD/aug-cc-pVTZ level of theory. The unimolecular rate constants obtained from the RRKM and VTST calculations for collision energies in the $0-33.2 \mathrm{~kJ} \mathrm{~mol}^{-1}$ interval were used to compute the branching ratios for the formation of the $\mathrm{SiO}_{2}+\mathrm{H}$ and $\mathrm{SiO}+\mathrm{OH}$ products within the reaction scheme illustrated in Fig. 3 employing the steady-state approximation. It should be noted that because of very-high-energy content in the intermediate [i2] and relatively low activation energies required for isomerization and dissociation steps, the product branching ratios appeared to be insensitive to the collision energy within the considered range.

Data availability. The data that support the findings of the current research are available from the corresponding authors upon request.

Received: 1 June 2017 Accepted: 24 January 2018

Published online: 22 February 2018

\section{References}

1. Ziurys, L. M. The chemistry in circumstellar envelopes of evolved stars: following the origin of the elements to the origin of life. Proc. Natl Acad. Sci. USA 103, 12274-12279 (2006).

2. Goumans, T. P. M. \& Bromley, S. T. Efficient nucleation of stardust silicates via heteromolecular homogeneous condensation. Mon. Not. R. Astron. Soc. 420, 3344-3349 (2012).

3. Henning, T. Cosmic silicates. Annu. Rev. Astron. Astrophys. 48, 21-46 (2010).

4. Tielens, A. G. G. M. in Nature's Nanostructures (eds Amanda S. Barnard \& Haibo Guo) 361-384 (Pan Stanford Publishing Pte. Ltd., 2012).

5. Jones, A. P. \& Nuth, J. A. III Dust destruction in the ISM: a re-evaluation of dust lifetimes. Astron. Astrophys. 530, A44 (2011).

6. Wakelam, V. et al. Reaction networks for interstellar chemical modelling: improvements and challenges. Space Sci. Rev. 156, 13-72 (2010).

7. Ehrenfreund, P. \& Charnley, S. B. Organic molecules in the interstellar medium, comets, and meteorites: a voyage from dark clouds to the early earth. Annu. Rev. Astron. Astrophys. 38, 427-483 (2000).

8. Abplanalp, M. J. et al. A study of interstellar aldehydes and enols as tracers of a cosmic ray-driven nonequilibrium synthesis of complex organic molecules. Proc. Natl. Acad. Sci. USA 113, 7727-7732 (2016).

9. Draine, B. T. \& Salpeter, E. E. Destruction mechanisms for interstellar dust. Astrophys. J. 231, 438-455 (1979).

10. Dwek, E. \& Scalo, J. M. The evolution of refractory interstellar grains in the solar neighborhood. Astrophys. J. 239, 193-211 (1980).

11. Jones, A. P., Tielens, A. G. G. M., Hollenbach, D. J. \& McKee, C. F. Grain destruction in shocks in the interstellar medium. Astrophys. J. 433, 797-810 (1994).

12. Zhukovska, S., Dobbs, C., Jenkins, E. B. \& Klessen, R. S. Modeling Dust Evolution in Galaxies with a Multiphase, Inhomogeneous ISM. Astrophys. J. 831, 147 (2016).

13. Michałowski, M. J. Dust production 680-850 million years after the Big Bang. Astron. Astrophys. 577, A80 (2015).

14. McKinnon, R., Torrey, P. \& Vogelsberger, M. Dust formation in Milky Waylike galaxies. Mon. Not. R. Astron. Soc. 457, 3775-3800 (2016).

15. Gail, H.-P. \& Sedlmayr, E. Mineral formation in stellar winds. I. Condensation sequence of silicate and iron grains in stationary oxygen rich outflows. Astron. Astrophys. 347, 594-616 (1999).

16. Reber, A. C. et al. Silicon oxide nanoparticles reveal the origin of silicate grains in circumstellar environments. Nano. Lett. 6, 1190-1195 (2006).

17. Reber, A. C. et al. From SiO molecules to silicates in circumstellar space: atomic structures, growth patterns, and optical signatures of $\mathrm{Si}_{\mathrm{n}} \mathrm{O}_{\mathrm{m}}$ clusters. ACS Nano 2, 1729-1737 (2008).

18. Gobrecht, D., Cherchneff, I., Sarangi, A., Plane, J. \& Bromley, S. Dust formation in the oxygen-rich AGB star IK Tauri. Astron. Astrophys. 585, A6 (2016).
19. Gail, H.-P., Wetzel, S., Pucci, A. \& Tamanai, A. Seed particle formation for silicate dust condensation by $\mathrm{SiO}$ nucleation. Astron. Astrophys. 555, A119 (2013).

20. Gail, H.-P., Scholz, M. \& Pucci, A. Silicate condensation in Mira variables. Astron. Astrophys. 591, A17 (2016).

21. Tielens, A. G. G. M. Interstellar depletions and the life cycle of interstellar dust. Astrophys. J. 499, 267-272 (1998).

22. Jones, A. P. Interstellar and circumstellar grain formation and survival. Philos Trans. R. Soc. Lond. A 359, 1961-1972 (2001).

23. Dwek, E. Iron: a key element for understanding the origin and evolution of interstellar dust. Astrophys. J. 825, 136 (2016).

24. Draine, B. T. Cosmic dust-near and far. ASP Conf. Ser. 414, 453-472 (2009)

25. Krasnokutski, S. A. et al. Formation of silicon oxide grains at low temperature Astrophys. J. 782, 15 (2014)

26. Rouillé, G., Jäger, C., Krasnokutski, S. A., Krebsz, M. \& Henning, T. Cold condensation of dust in the ISM. Faraday Discuss. 168, 449-460 (2014).

27. In the ISM, silylidyne radicals $(\mathrm{SiH})$ would react with molecular oxygen. In the laboratory, the reaction was conducted between D1-silylidyne and molecular oxygen to elucidate the atomic deuterium loss because of the difficulties in preparing silylidyne radicals.

28. Suto, M. \& Lee, L. C. Quantitative photoexcitation study of $\mathrm{SiH}_{4}$ in vacuum ultraviolet. J. Chem. Phys. 84, 1160-1164 (1986).

29. Itoh, U., Toyoshima, Y., Onuki, H., Washida, N. \& Ibuki, T. Vacuum ultraviolet absorption cross sections of $\mathrm{SiH}_{4}, \mathrm{GeH}_{4}, \mathrm{Si}_{2} \mathrm{H}_{6}$, and $\mathrm{Si}_{3} \mathrm{H}_{8}$. J. Chem. Phys. 85, 4867-4872 (1986).

30. Glenewinkel-Meyer, T., Bartz, J. A., Thorson, G. M. \& Crim, F. F. The vacuum ultraviolet photodissociation of silane at $125.1 \mathrm{~nm}$. J. Chem. Phys. 99, 5944-5950 (1993)

31. Avramov, P. V. et al. Potential energy surfaces of $\mathrm{Si}_{\mathrm{m}} \mathrm{O}_{\mathrm{n}}$ cluster formation and isomerization. J. Phys. Chem. A. 109, 6294-6302 (2005).

32. Vernon, M. F. Molecular-beam scattering, University of California at Berkeley (1983).

33. Linstrom, P. J. \& Mallard, W. NIST Chemistry WebBook; NISTStandard Reference Database No. 69,http://webbook.nist.gov (2001).

34. Kostko, O., Ahmed, M. \& Metz, R. B. Vacuum-ultraviolet photoionization measurement and ab initio calculation of the ionization energy of gas-phase $\mathrm{SiO}_{2}$. J. Phys. Chem. A. 113, 1225-1230 (2009).

35. Wang, L.-S., Wu, H., Desai, S. R., Fan, J. \& Colson, S. D. A photoelectron spectroscopic study of small silicon oxide clusters: $\mathrm{SiO}_{2}, \mathrm{Si}_{2} \mathrm{O}_{3}$, and $\mathrm{Si}_{2} \mathrm{O}_{4}$. J. Phys. Chem. 100, 8697-8700 (1996).

36. Levine, R. D. Molecular Reaction Dynamics. (Cambridge University Press, 2005).

37. Miller, W. B., Safron, S. A. \& Herschbach, D. R. Exchange reactions of alkali atoms with alkali halides: a collision complex mechanism. Discuss. Faraday Soc. 44, 108-122 (1967).

38. Kaiser, R. I., Ochsenfeld, C., Head-Gordon, M., Lee, Y. T. \& Suits, A. G. A combined experimental and theoretical study on the formation of interstellar $\mathrm{C}_{3} \mathrm{H}$ Isomers. Science 274, 1508-1511 (1996).

39. Hao, Y., Xie, Y. \& Schaefer, H. F. III Features of the potential energy surface for the $\mathrm{SiO}+\mathrm{OH} \rightarrow \mathrm{SiO}_{2}+\mathrm{H}$ reaction: relationship to oxygen isotopic partitioning during gas phase $\mathrm{SiO}_{2}$ formation. RSC Adv. 4, 47163-47168 (2014).

40. Darling, C. L. \& Schlegel, H. B. Heats of formation of $\mathrm{SiH}_{\mathrm{n}} \mathrm{O}$ and $\mathrm{SiH}_{\mathrm{n}} \mathrm{O}_{2}$ calculated by ab initio molecular orbital methods at the G-2 level of theory. J. Phys. Chem. 97, 8207-8211 (1993).

41. Adamovic, I. \& Gordon, M. S. Potential energy surfaces for the reactions $\mathrm{Si}$ $+\mathrm{O}_{2}$. J. Phys. Chem. A. 108, 8395-8399 (2004).

42. Gómez Martín, J. C. \& Plane, J. M. C. Kinetic studies of atmospherically relevant silicon chemistry. Part III: Reactions of $\mathrm{Si}^{+}$and $\mathrm{SiO}^{+}$with $\mathrm{O}_{3}$, and $\mathrm{Si}$ ${ }^{+}$with $\mathrm{O}_{2}$. Phys. Chem. Chem. Phys. 13, 3764-3774 (2011).

43. Krasnokutski, S. A. \& Huisken, F. Oxidative reactions of silicon atoms and clusters at ultralow temperature in helium droplets. J. Phys. Chem. A. 114, 13045-13049 (2010).

44. Langer, W. D. \& Glassgold, A. E. Silicon chemistry in interstellar clouds. Astrophys. J. 352, 123-131 (1990).

45. Yamashiro, R., Matsumoto, Y. \& Honma, K. Reaction dynamics of $\mathrm{Si}\left({ }^{3} \mathrm{P}_{\mathrm{J}}\right)$ $+\mathrm{O}_{2} \rightarrow \mathrm{SiO}\left(\mathrm{X}^{1} \Sigma^{+}\right)+\mathrm{O}$ studied by a crossed-beam laser-induced fluorescence technique. J. Chem. Phys. 128, 084308 (2008).

46. Dayou, F. et al. A comparative study of the $\mathrm{Si}+\mathrm{O}_{2} \rightarrow \mathrm{SiO}+\mathrm{O}$ reaction dynamics from quasiclassical trajectory and statistical based methods. J. Chem. Phys. 128, 174307 (2008).

47. Takakuwa, Y., Nihei, M., Horie, T. \& Miyamoto, N. Thermal oxidation mechanism based on formation and diffusion of volatile $\mathrm{SiO}$ molecules. $J$. Non-Cryst. Solids 179, 345-353 (1994).

48. Gómez Martín, J. C., Blitz, M. A. \& Plane, J. M. C. Kinetic studies of atmospherically relevant silicon chemistry. Part II: silicon monoxide reactions. Phys. Chem. Chem. Phys. 11, 10945-10954 (2009). 
49. Bocherel, $\mathrm{P}$. et al. Ultralow-temperature kinetics of $\mathrm{CH}\left(\mathrm{X}^{2} \Pi\right)$ reactions: rate coefficients for reactions with $\mathrm{O}_{2}$ and $\mathrm{NO}(\mathrm{T}=13-708 \mathrm{~K})$, and with $\mathrm{NH}_{3}$ ( $\mathrm{T}=23-295$ K). J. Phys. Chem. 100, 3063-3069 (1996).

50. Georgievskii, Y. \& Klippenstein, S. J. Long-range transition state theory. J. Chem. Phys. 122, 194103 (2005).

51. Nemoto, M., Suzuki, A., Nakamura, H., Shibuya, K. \& Obi, K. Electronic quenching and chemical reactions of $\mathrm{SiH}$ radicals in the gas phase. Chem. Phys. Lett. 162, 467-471 (1989).

52. Geballe, T. R. \& Oka, T. A key molecular ion in the Universe and in the laboratory. Science 312, 1610-1612 (2006).

53. Chen, J.-H. et al. Herschel HIFI observations of $\mathrm{O}_{2}$ toward Orion: special conditions for shock enhanced emission. Astrophys. J. 793, 111 (2014).

54. Larsson, B. et al. Molecular oxygen in the $\rho$ Ophiuchi cloud. Astron. Astrophys. 466, 999-1003 (2007).

55. Liseau, R. et al. Multi-line detection of $\mathrm{O}_{2}$ toward $\rho$ Ophiuchi A. Astron. Astrophys 541, A73 (2012).

56. Goldsmith, P. F. et al. Herschel measurements of molecular oxygen in Orion. Astrophys. J. 737, 96 (2011).

57. Elsila, J., Allamandola, L. J. \& Sandford, S. A. The $2140 \mathrm{~cm}^{-1}$ (4.673 microns) solid CO band: The case for interstellar $\mathrm{O}_{2}$ and $\mathrm{N}_{2}$ and the photochemistry of nonpolar interstellar ice analogs. Astrophys. J. 479, 818 (1997).

58. Vandenbussche, B. et al. Constraints on the abundance of solid $\mathrm{O}_{2}$ in dense clouds from ISO-SWS and ground-based observations. Astron. Astrophys. 346, L57-L60 (1999).

59. Bieler, A. et al. Abundant molecular oxygen in the coma of comet $67 \mathrm{P} /$ Churyumov-Gerasimenko. Nature 526, 678-681 (2015)

60. Taquet, V., Furuya, K., Walsh, C. \& van Dishoeck, E. F. A primordial origin for molecular oxygen in comets: a chemical kinetics study of the formation and survival of $\mathrm{O}_{2}$ ice from clouds to discs. Mon. Not. R. Astron. Soc. 462, S99-S115 (2016)

61. Pettersson, L. G. M. \& Langhoff, S. R. Theoretical electric dipole moments of $\mathrm{SiH}, \mathrm{GeH}$ and SnH. Chem. Phys. Lett. 125, 429-432 (1986).

62. Schilke, P., Benford, D. J., Hunter, T. R., Lis, D. C. \& Phillips, T. G. A line survey of Orion-KL from 607 to $725 \mathrm{GHz}$. Astrophys. J. Suppl. Ser. 132, 281-364 (2001).

63. Choy, K. L. Chemical vapour deposition of coatings. Prog. Mater. Sci. 48, 57-170 (2003).

64. Jasinski, J. M. \& Gates, S. M. Silicon chemical vapor deposition one step at a time: fundamental studies of silicon hydride chemistry. Acc. Chem. Res. 24, 9-15 (1991).

65. Pierson, H. O. Handbook of Chemical Vapor Deposition: Principles, Technology and Applications. (William Andrew, 1999).

66. Wolf, S. \& Tauber, R. N. Silicon Processing for the VLSI Era, Process Technology. 2nd edn, Vol. 1 (Lattice Press, 2000).

67. Adams, A. C., Alexander, F. B., Capio, C. D. \& Smith, T. E. Characterization of plasma-deposited silicon dioxide. J. Electrochem. Soc. 128, 1545-1551 (1981).

68. Batey, J. \& Tierney, E. Low-temperature deposition of high-quality silicon dioxide by plasma-enhanced chemical vapor deposition. J. Appl. Phys. 60, 3136-3145 (1986).

69. Jasinski, J. M., Becerra, R. \& Walsh, R. Direct kinetic studies of silicon hydride radicals in the gas phase. Chem. Rev. 95, 1203-1228 (1995).

70. Birnstiel, T., Fang, M. \& Johansen, A. Dust evolution and the formation of planetesimals. Space Sci. Rev. 205, 41-75 (2016).

71. Kaiser, R. I. et al. Untangling the chemical evolution of Titan's atmosphere and surface-from homogeneous to heterogeneous chemistry. Faraday Discuss. 147, 429-478 (2010).

72. Parker, D. S. N. et al. On the formation of silacyclopropenylidene $\left(\mathrm{c}-\mathrm{SiC}_{2} \mathrm{H}_{2}\right)$ and its role in the organosilicon chemistry in the interstellar medium. Astrophys. J. 770, 33 (2013).

73. Bauer, W., Becker, K. H., Düren, R., Hubrich, C. \& Meuser, R. Radiative lifetime measurements of $\mathrm{SiH}\left(\mathrm{A}^{2} \Delta\right)$ by laser-induced fluorescence. Chem. Phys. Lett. 108, 560-561 (1984).

74. Yang, T. et al. Combined experimental and theoretical study on the formation of the elusive 2-methyl-1-silacycloprop-2-enylidene molecule under single collision conditions via reactions of the silylidyne radical $\left(\mathrm{SiH} ; \mathrm{X}^{2} \Pi\right)$ with Allene $\left(\mathrm{H}_{2} \mathrm{CCCH}_{2} ; \mathrm{X}^{1} \mathrm{~A}_{1}\right)$ and D4-Allene $\left(\mathrm{D}_{2} \mathrm{CCCD}_{2} ; \mathrm{X}^{1} \mathrm{~A}_{1}\right)$. J. Phys. Chem. A. 119, 12562-12578 (2015).

75. Weiss, P. S. The reaction dynamics of electronically excited alkali atoms with simple molecules, University of California at Berkeley (1986).
76. Kaiser, R. I. et al. PAH formation under single collision conditions: reaction of phenyl radical and 1,3-butadiene to form 1,4-dihydronaphthalene. J. Phys. Chem. A. 116, 4248-4258 (2012).

77. Adler, T. B., Knizia, G. \& Werner, H.-J. A simple and efficient CCSD(T)-F12 approximation. J. Chem. Phys. 127, 221106 (2007)

78. Dunning, Jr,T. H. Gaussian basis sets for use in correlated molecular calculations. I. The atoms boron through neon and hydrogen. J. Chem. Phys. 90, 1007-1023 (1989).

79. Peterson, K. A., Adler, T. B. \& Werner, H.-J. Systematically convergent basis sets for explicitly correlated wave functions: the atoms $\mathrm{H}, \mathrm{He}, \mathrm{B}-\mathrm{Ne}$, and $\mathrm{Al}$ Ar. J. Chem. Phys. 128, 084102 (2008).

80. Celani, P. \& Werner, H.-J. Multireference perturbation theory for large restricted and selected active space reference wave functions. J. Chem. Phys. 112, 5546-5557 (2000).

81. Werner, H.-J. et al. Molpro, Version 2010.1, A Package of Ab Initio Programs. (TTI GmbH, Stuttgart, Germany 70173, 2010).

82. Kislov, V. V., Nguyen, T. L., Mebel, A. M., Lin, S. H. \& Smith, S. C. Photodissociation of benzene under collision-free conditions: an ab initio/ Rice-Ramsperger-Kassel-Marcus Study. J. Chem. Phys. 120, 7008-7017 (2004).

83. Truhlar, D. G. \& Garrett, B. C. Variational transition state theory. Annu. Rev Phys. Chem. 35, 159-189 (1984).

84. Truhlar, D. G., Isaacson, A. D. \& Garrett, B. C. in Theory of Chemical Reaction Dynamics Vol. 4 (ed M. Baer) 65-137 (CRC Press: Boca Raton, FL, U. S. A., 1985).

85. Chai, J.-D. \& Head-Gordon, M. Long-range corrected hybrid density functionals with damped atom-atom dispersion corrections. Phys. Chem. Chem. Phys. 10, 6615-6620 (2008).

\section{Acknowledgements}

We thank the National Science Foundation (NSF) for support under award CHE1360658. Astrophysics at Queen's University Belfast is supported by a grant, ST/ P000321/1, from the Science and Technology Facilities Council (UK).

\section{Author contributions}

T.Y., A.M.T., and B.B.D. carried out the experimental measurements; T.Y. performed the data analysis; A.M.M. carried out the theoretical analysis; T.J.M. carried out the astronomical discussion; T.Y., R.I.K. and A.M.M. wrote the paper; and T.Y. and R.I.K. supervised the study.

\section{Additional information}

Supplementary Information accompanies this paper at https://doi.org/10.1038/s41467018-03172-5.

Competing interests: The authors declare no competing financial interests.

Reprints and permission information is available online at http://npg.nature.com/ reprintsandpermissions/

Publisher's note: Springer Nature remains neutral with regard to jurisdictional claims in published maps and institutional affiliations.

Open Access This article is licensed under a Creative Commons Attribution 4.0 International License, which permits use, sharing, adaptation, distribution and reproduction in any medium or format, as long as you give appropriate credit to the original author(s) and the source, provide a link to the Creative Commons license, and indicate if changes were made. The images or other third party material in this article are included in the article's Creative Commons license, unless indicated otherwise in a credit line to the material. If material is not included in the article's Creative Commons license and your intended use is not permitted by statutory regulation or exceeds the permitted use, you will need to obtain permission directly from the copyright holder. To view a copy of this license, visit http://creativecommons.org/ licenses/by/4.0/

(C) The Author(s) 2018 